\title{
ENZYMATIC FUEL CELLS
}

\section{Improving power and stability \\ Energy Environ. Sci. 10, 1966-1982 (2017)}

$\mathrm{H}_{2} / \mathrm{O}_{2}$ enzymatic fuel cells (EFCs) offer a sustainable means of producing electricity through $\mathrm{H}_{2}$ oxidation and $\mathrm{O}_{2}$ reduction via enzyme biocatalysts. While EFCs may have economic benefits compared to Pt-based fuel-cell technologies, many challenges exist for practical applications. One major problem is that only a small fraction of enzymes effectively participates in the current generation, which is a main reason why the power density of EFCs is often small. Furthermore, it is difficult to detect electroactive enzymes because of the presence of their insulating protein shells as well as their usually low electrode surface coverage. Another critical issue is the enzyme stability, as contact with a gaseous phase or drying would lead to enzyme deactivation. Now, Ievgen Mazurenko, Elisabeth Lojou and colleagues in France design a high-power thermostable EFC, and quantify the active enzyme molecules participating in the electrocatalysis.

In their EFC, the researchers immobilize enzyme biocatalysts in porous electrodes consisting of carbon felt modified by carbon nanotubes. The hierarchical structure of the carbon electrodes allows fine-tuning of their porosity and thickness to approach optimal enzyme distribution and mass transport. A power-density of $1.0 \mathrm{~mW} \mathrm{~cm}^{-2}$ is achieved, which is among the highest values reported for EFCs, with only 5\% power loss during 17 hours of operation at room temperature. The good stability is largely ascribed to the porous carbon matrix, which shields the enzyme molecules from reactions with harmful oxygen species. Importantly, the carbon matrix also allows high enzyme loading, which enables the researchers to perform enzyme quantification. Using adsorption isotherms and electrochemical measurements, the researchers show that $10-15 \%$ of the total quantity of adsorbed enzymes contributes to the electrocatalysis. This indicates that there is plenty of room for enhancing the amount of active enzymes to further improve the performance of EFCs.

Changjun Zhang

Published online: 10 October 2017

DOI: 10.1038/s41560-017-0021-z 